\title{
Direct or Collateral Liver Damage in SARS-CoV-2-Infected Patients
}

\author{
Maria J. Lizardo-Thiebaud, MD, MsC ${ }^{1, *}$ Eduardo Cervantes-Alvarez, MD²,3, \\ Nathaly Limon-de la Rosa, BS ${ }^{2}$ Farid Tejeda-Dominguez, $\mathrm{PhD}^{1}$ Mildred Palacios-jimenez, MD ${ }^{2,4}$ \\ Osvely Méndez-Guerrero, $\mathrm{MSc}^{2}$ Marco Delaye-Martinez, MD ${ }^{2,3}$ Fatima Rodriguez-Alvarez, MD ${ }^{2,4}$ \\ Beatriz Romero-Morales, BS ${ }^{2}$ Wei-Hui Liu, MD, PhD ${ }^{5}$ Christene A. Huang, $\mathrm{PhD}^{6}$ \\ David Kershenobich, MD, $\mathrm{PhD}^{2}$ Nalu Navarro-Alvarez, MD, $\mathrm{PhD}^{1,2,6, *}$
}
${ }^{1}$ Department of Molecular Biology, Universidad Panamericana, School of Medicine, Campus México, Mexico City
2 Department of Gastroenterology, Instituto Nacional de Ciencias Médicas y Nutrición Salvador Zubirán, Mexico City, Mexico
3 PECEM, Facultad de Medicina, Universidad Nacional Autónoma de México, Mexico City, Mexico
${ }^{4}$ Department of Medicine, Universidad Veracruzana, Veracruz, Mexico
${ }^{5}$ Department of Gastroenterology and Hepatology, Sichuan Academy of Medical Sciences and Sichuan Provincial People's Hospital, Chengdu, Sichuan Province, China
${ }^{6}$ Department of Surgery, University of Colorado Anschutz Medical Campus, Denver, Colorado

Semin Liver Dis 2020;40:321-330.

Address for correspondence Nalu Navarro-Alvarez, MD, PhD, Instituto Nacional de Ciencias Médicas y Nutrición Salvador Zubirán, Vasco de Quiroga \#15, Tlalpan, 14080, CDMX, Mexico (e-mail: nalu.navarroa@incmnsz.mx).

\section{Abstract \\ Keywords \\ - liver \\ - SARS-CoV-2 \\ - COVID-19 \\ - ACE2 \\ - inflammasome}

Liver injury can result from severe acute respiratory syndrome coronavirus 2 (SARS-CoV-2) infection with more than one-third of COVID-19 patients exhibiting elevated liver enzymes. Microvesicular steatosis, inflammation, vascular congestion, and thrombosis in the liver have been described in autopsy samples from COVID-19 patients. Several factors, including direct cytopathic effect of the virus, immune-mediated collateral damage, or an exacerbation of preexisting liver disease may contribute to liver pathology in COVID-19. Due to its immunological functions, the liver is an organ likely to participate in the viral response against SARS-CoV-2 and this may predispose it to injury. A better understanding of the mechanism contributing to liver injury is needed to develop and implement early measures to prevent serious liver damage in patients suffering from COVID-19. This review summarizes current reports of SARS-CoV-2 with an emphasis on how direct infection and subsequent severe inflammatory response may contribute to liver injury in patients with and without preexisting liver disease.
In mid-December 2019, in Wuhan, in the Hubei province of China, a cluster of five patients with severe acute respiratory syndrome (SARS) was admitted to a hospital and diagnosed with pneumonia of unknown etiology. According to the epidemiological follow-up, the initial cases were linked to a seafood and wet animal wholesale market. ${ }^{1}$ On February 12 ,

Contributed equally to this manuscript.
2020, the World Health Organization (WHO) named the new coronavirus severe acute respiratory syndrome coronavirus 2 (SARS-CoV-2) and the disease coronavirus disease (COVID)-19, which has since been classified as a pandemic. As of June 30, 2020, there were more than 10.1 million cases and 503,862 deaths, ${ }^{2}$ with the United States, Russia, United Kingdom, Brazil, Spain, India, and Italy being the most affected countries by the pandemic. A higher prevalence 
has been reported in adults aged 18 to 64 years, representing approximately $70 \%$ of the infected population. ${ }^{3}$ However, individuals 65 years or older have the highest case fatality rates. $^{4,5}$

Lethality due to COVID-19 has been associated with an exacerbated inflammatory response, namely, a cytokine storm characterized by high circulating levels of proinflammatory cytokines and chemokines, ${ }^{6,7}$ a phenomenon that is not uncommon to the previously identified coronaviruses, SARS$\mathrm{CoV}$ and Middle East respiratory syndrome-coronavirus (MERS-CoV) which appeared in 2002 and 2012, respectively. ${ }^{8}$ In both cases, severe disease is known to manifest as a severe acute respiratory distress syndrome (ARDS), and in many cases liver damage may occur. Manifestations of hepatic injury caused by either virus are similar, and are evident mainly due to elevated transaminase levels, observed in $60 \%$ in the case of SARS-CoV-infected patients. ${ }^{9}$ Liver histological changes in SARS-CoV include mild to moderate lobular inflammation and cells in mitosis, ${ }^{9}$ whereas in MERS moderate steatosis, scattered calcifications, mild portal tract, and lobular lymphocytic inflammation are reported. ${ }^{10}$

This novel COVID-19 disease parallels many aspects of the previous coronavirus epidemics, including the importance of the severe inflammatory response syndrome (SIRS) which contributes to ARDS, and eventually progresses to multiorgan failure (MOF). In this process, the whole system is at risk, as other organs, besides the lungs, suffer from a bystander effect, and the liver is no exception. This inflammatory process is potentiated by the continuous activation of the inflammasome, ${ }^{8}$ which is induced not only by the release of damage-associated molecular patterns (DAMPs) from injured tissues but also by virulence factors unique to these coronaviruses. SARS-CoV-2, with its particular and apparently more successful infectious capacity, has also proven to induce reactive hepatitis, as evidenced by liver function test (LFT) abnormalities, which correlate with an increased risk of severe disease. ${ }^{11,12}$ Additionally, it is now known that liver damage also occurs due to direct infection of the hepatocytes, and not only as a consequence of the sustained inflammatory response. In this review, we will summarize current reports of SARS-CoV-2, its infection mechanisms and contribution to SIRS, as well as possible liver dysfunction in patients with and without preexisting liver disease.

\section{SARS-CoV-2 Infection Mechanisms}

The genome of SARS-CoV-2 comprises $29.9 \mathrm{~kb}$ and shares 79.6\% sequence identity to SARS-CoV and 50\% genome sequence homology with MERS-CoV. ${ }^{13}$ Moreover, it has $96 \%$ genome identity to a bat coronavirus, suggesting it originated in bats. ${ }^{14}$

SARS-CoV-2 is a beta-coronavirus of the family of CoVs and the causal agent of COVID-19. Similar to SARS-CoV, SARS-CoV-2 mediates its entry to the target cell through binding to the angiotensin-converting enzyme 2 (ACE2) functional receptor, ${ }^{13}$ a type I membrane protein found in several tissues including lungs, heart, kidneys, intestine, and liver. ${ }^{13,15}$ Thus, the tropism of virus infection is determined by tissue expression and distribution of the receptor. ${ }^{16}$

As with all CoVs, SARS-CoV-2 has a positive singlestranded RNA genome which contains two genomic open reading frames (ORFs: ORF1a and ORF1b) and nine ORFs that are part of the sub-genomic RNA. The translation of ORF1a and ORF1b encodes a polyprotein containing the replicase which has proteases that cleave the polyprotein to release nonstructural proteins (nsp) to form the replicase-transcriptase complex. The other nine ORFs code for conserved structural proteins like the spike protein $(S)$, the envelope protein $\mathrm{E}$, the membrane protein $(\mathrm{M})$, the nucleocapsid protein, and at least six accessory proteins (3a, 6, 7a, 7b, 8, and 10). ${ }^{17}$

CoVs initiate their infection when the $\mathrm{S}$ protein is cleaved by host proteases like cathepsin $\mathrm{L}$ and the transmembrane protease serine 2 (TMPRSS2) in the case of SARS-CoV-2, enabling the virus the ability of attachment, fusion, and entry. This is mediated by binding to the host cell through the receptor-binding domain (RBD) in its S1 subunit ${ }^{18}$ which binds directly to the peptidase domain of ACE $2^{15}$ and by fusing the viral and host membranes through its S2 subunit. ${ }^{18}$ Its entry can also be mediated by endocytosis which is driven by PIKfyve and two pore segment channel 2 (TPC2). ${ }^{19,20}$ Once inside the host cell cytoplasm, the replicase-transcriptase complex is assembled and generates new genomic RNA and sub-genomic RNA, used as a mRNA mold. The structural proteins are synthesized by the endoplasmic reticulum and transported to the cytosol where the virions are built and finally transported to the cell membrane to be exocytosed. ${ }^{11}$

Remarkably, the S protein in SARS-CoV-2 is expressed as a proprotein convertase (PPC), which is proteolytically cleaved by cytosolic furin, inducing preactivation of the $S$ protein. Once activated, it exposes the S1 and S2 subunit, thereby enhancing the entry of SARS-CoV-2. Thus, it is the combination of these proteases that favors the processing of the $S$ protein and the virus internalization in susceptible cells. It is important to note that in cells with low cathepsin L and/or low expression of TMPRSS2, the furin preactivation process can promote SARS-CoV-2 infection. ${ }^{21}$ Also, it has been demonstrated that the S1 CTD (C terminal domain) binds to human ACE2 (hACE2) by its RBD and that this interaction happens between the external subdomain of SARS CoV-2CTD that binds with the hACE2 N-terminal domain (NTD) subdomain I. ${ }^{22}$ Interestingly, this interaction is four times higher than that observed for the homologous regions of SARS-CoV S protein. This is because the RBD is more compact and presents more atoms that interact with the hACE2 NTD. ${ }^{23}$ Importantly, this RBD undergoes conformational changes between standing-up/-down positions that favor receptor binding or immune evasion, respectively. Surprisingly, the RBD from SARS-CoV-2 is more frequently found to be in the standing-down position, which prevents immune recognition by the host, but at the same time affects RBD availability for binding. However, this is compensated with the high affinity for its receptor and a preactivation process facilitating SARS-CoV-2 entry to the host, which combined 
with the low immune response produced, increases SARSCoV-2 pathogenicity. ${ }^{21}$

\section{Physiopathology of the Inflammatory Response in SARS-CoV-2-Infected Patients}

While we currently do not completely understand COVID-19 pathophysiology, we know now that SARS-CoV-2 causes similar clinical characteristics as those manifested in SARS-CoV and MERS-CoV infections, ${ }^{6}$ including severe respiratory symptoms.

The clinical presentation of SARS-CoV-2-infected patients mainly consists of fever, fatigue, dry cough, anorexia, myalgia, and gastrointestinal (GI) symptoms such as diarrhea, nausea, vomiting, and abdominal pain. ${ }^{12}$ However, loss of smell and taste and less frequently dermatological findings such as maculopapular plaques, urticaria, vesicles, and livedo reticularis ${ }^{24}$ have also been reported.

It is suggested that since ACE2 is mostly expressed in type II alveolar cells, the injury to these cells explains the severe alveolar damage and minimal upper airway symptoms ${ }^{16}$ observed in these patients, seen as bilateral ground-glass opacity and subsegmental areas of consolidation by chest computed tomography. ${ }^{6}$

The systemic inflammatory response syndrome ${ }^{25}$ is a cardinal feature of lung disease caused by SARS-CoV-2, as evidenced by elevated levels of CRP, procalcitonin, and serum amyloid A. ${ }^{26}$ Severe SIRS culminates in respiratory failure due to ARDS, which along with secondary hemophagocytic lymphohistiocytosis are considered important causes of a hyperinflammatory state that can progress to MOF. ${ }^{7}$

Indeed, COVID-19 infection is characterized by a cytokine storm with high circulating levels of proinflammatory cytokines and chemokines including interleukin (IL)- $1 \beta$, interferon (IFN)- $r$, inducible protein (IP)-10, and MCP-1, similarly with SARS-CoV and MERS patients. ${ }^{6}$ Importantly, the bronchoalveolar lavage (BAL) fluid of ARDS nonsurvivors has elevated and persistent levels of IL-1 $\beta$, IL-6, IL-8, and tumor necrosis factor (TNF)- $\alpha$ in association with increased endothelial permeability, elevated BAL/plasma ratios of all of these cytokines, suggests a pulmonary origin. ${ }^{27}$ There is evidence pointing to the activated inflammasome as one of the critical mediators of this cytokine storm, as increased levels of mRNA transcripts of caspase-1, IL-1 $\beta$, and IL-18 have been found in the peripheral blood of these patients. ${ }^{28}$ Thus, a similar or more potent inflammatory response can be expected in COVID-19, as explained by the fact that coronaviruses such as SARS-CoV, MERS, and SARS-CoV-2 encode activators of the NOD-like receptor pyrin domain-containing 3 (NLRP3) inflammasome, the most studied multimeric protein complex that amplifies inflammatory signals.

One of those activators in SARS-CoV-2 is ORF3a, which induces NF-kB activation, chemokine production, Golgi fragmentation, ER stress, and cell death. ${ }^{29}$ In addition, it promotes inflammasome assembly via ubiquitination of apoptosis-associated speck-like protein containing CARD (ASC), ${ }^{30}$ thus promoting pro-IL-1 $\beta$ gene transcription. Likewise, $\mathrm{E}$ protein also activates the NLRP3 inflammasome. ${ }^{8}$ Besides being a structural protein, $\mathrm{E}$ protein is an ion channel, exerting its activity in the membrane of the ER. The release of $\mathrm{Ca}^{2+}$ from the ER contributes to the activation of the inflammasome complex, leading to the release of proinflammatory cytokines such as TNF-a, IL-1, and IL-6. The accumulation of these cytokines promotes an exacerbated proinflammatory response that contributes to the SIRS, whose consequences may be lethal. ${ }^{25,31}$

Recently, inoculation of murine coronavirus mouse hepatitis virus A-59 (MHV-A59) in mice has been proposed as an effective animal model to study ARDS caused by SARS-CoV and MERS infections, which would avoid the necessary high level of containment required otherwise. After intranasal inoculation, MHV-A59 was found to induce pathologic features of acute lung injury which were additionally associated with increased levels of IP-10, IFN- $\gamma$, TNF- $\alpha$, and IL-1 $\beta$, suggestive of the elevated inflammatory response expected in these infections, and interestingly a suppressed antiviral type I IFN response as well. ${ }^{32} \mathrm{~A}$ study of inflammasome activation with a similar mouse model (intracranial inoculation of MHV-A59) showed results consistent with a protective role, as infected caspase-1 knockout (KO) mice had reduced survival ( 40 vs. $90 \%$ in WT) in addition to increased viral replication in the liver and spleen. Furthermore, IL-18 KO mice had a much lower survival, even though the infective dose was smaller (10 vs. $90 \%$ in WT); elevated viral load and poor viral clearance were also seen in the liver of these mice, all of which were associated with impaired IFN- $\gamma$ production. However, results from IL-1 KO mice suggested a pathological role as survival tended to be higher than in WT mice, although a model-specific effect could not be ruled out. ${ }^{33}$ These findings hint to the complexity of the immune system, whose response depends on the type and intensity of the stimuli, with the possibility of being overwhelmed if persistent.

An additional physiopathological component that contributes to the inflammatory response, including the activation of the inflammasome, is the DAMP released as part of the bystander effect related to the multiorgan involvement inherent to ARDS. Indeed, IL-33, a molecule released after epithelial cell damage or necrosis, is found at high levels in the serum of ARDS patients. ${ }^{34}$ Likewise, alveolar epithelial cells are known to be activated by S100A8 via TLR4 promoting neutrophil recruitment to the lungs, ${ }^{35}$ while mitochondrial DAMPs (DNA and proteins) on the other hand increase systemic endothelial permeability by upregulating the expression of HMGB1, another important DAMP, and also activate neutrophils and enhance their adherence to the endothelium. ${ }^{36}$ This severe immune response also leads to an impaired coagulation cascade, predisposing these patients to the formation of microthrombosis, disseminated intravascular coagulation, and $\mathrm{MOF}^{31}$

\section{Direct or Collateral Liver Damage in SARS-CoV-2}

The liver is a key organ in all metabolic processes. It harbors the body's largest macrophage pool and its parenchymal and nonparenchymal cells (hepatic stellate cells and liver sinusoidal endothelial cells) are primed to sense and activate immune responses via pathogen recognition receptors (PRR) such as toll-like receptor (TLR) $4 .{ }^{37}$ This is of great 


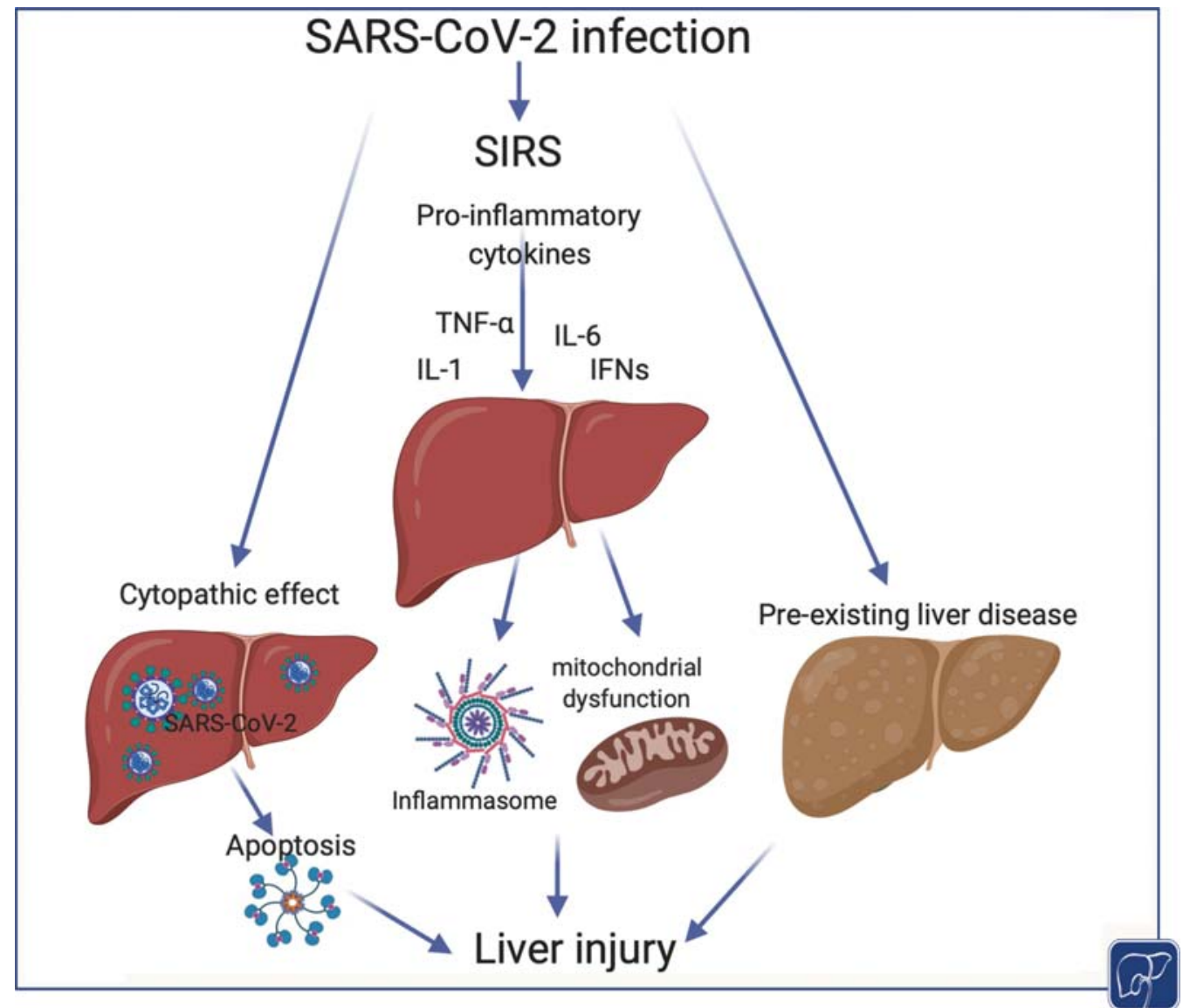

Fig. 1 Liver injury in COVID-19. When the virus infects the liver parenchyma, its virulence factors promote apoptosis. With the establishment of SIRS, proinflammatory cytokines make target organs susceptible, including the liver. They mainly cause mitochondrial dysfunction but can also activate the inflammasome via DAMPs. With these two processes put together, a liver with low functional reserve due to a chronic injury is vulnerable to damage either by the virus itself or by the inflammatory state. IFN, interferon; IL, interleukin; SARS-CoV-2, severe acute respiratory syndrome coronavirus 2; SIRS, severe inflammatory response syndrome; TNF, tumor necrosis factor. (BioRender was used to make the figures.)

importance owing to the liver's dual blood supply, of which a significant number of antigens arrive from the gut via the portal vein. In this sense, the liver is an organ likely to participate in the viral response against SARS-CoV-2, whose known functional receptor, ACE2, has been detected in cholangiocytes, $^{38}$ vascular endothelium, and perivenular hepatocytes. $^{39}$

Liver injury has been observed in SARS-CoV-2-infected patients ${ }^{40}$ with an incidence of $36 \%$ (range, $21-52 \%$ ), ${ }^{41}$ and liver samples obtained from autopsies of COVID-19 patients show microvesicular steatosis and inflammation. ${ }^{42-44}$ While the specific mechanisms of liver injury have not been fully identified, the following ones have been proposed and are summarized in -Fig. 1.

\section{Direct Cytopathic Effects of SARS-CoV-2 on Parenchymal Cells}

In theory, all viruses have a certain degree of cytopathic effects, as they hijack the cell's translational machinery. The extent to which cells respond to the viruses varies according to their own physiological transcriptional activity.
Among the parenchymal cells composing the liver, cholangiocytes have been reported to express higher ACE2, ${ }^{38}$ and therefore could provide access for the virus to gain entry into the liver and cause damage. In a 3D culture model of human cholangiocytes, 2.92\% of the cells expressed ACE2 and 51.54\% of the cells expressed TMPRSS2 after single-cell RNA sequencing, which was also confirmed by immunostaining. Interestingly, $68 \%$ of those ACE2 + cells coexpress TMPRSS2, which makes cholangiocytes an easy target for SARS-CoV-2 infection. Indeed, not only the expression of SARS-CoV-2 nucleocapsid $(\mathrm{N})$ protein inside cholangiocytes was demonstrated in this in vitro system 24 hours after infection, but an increase in viral load by genomic RNA analysis was also observed. ${ }^{45}$ More importantly, the infection of cholangiocytes by SARS-CoV-2 altered their bile acid (BA) transporting function reducing the expression of the apical sodium-dependent BA transporter and the cystic fibrosis transmembrane regulator. ${ }^{45,46}$ Viral load in these organoids decreased after 48 hours and was associated with a high expression of genes related to apoptosis, suggesting that SARS-CoV-2 infection induces cholangiocyte death. ${ }^{46}$ Cholestasis has been recently reported in 7 out of 11 
autopsies performed in COVID-19 patients at a medical center in Austria, with nuclear pleomorphism in cholangiocytes and ductular proliferation. ${ }^{47}$

Despite this evidence, COVID-19 patients with liver damage do not exhibit severe alterations in gamma-glutamyl transferase (GGT) or alkaline phosphatase (ALP) levels. ${ }^{48}$ Instead, moderate elevations of aspartate aminotransferase (AST) and alanine aminotransferase (ALT) are observed, suggesting a direct effect on hepatocytes.

ACE2 expression in hepatocytes of normal liver tissue is low and has been observed only in occasional perivenular hepatocytes. ${ }^{39}$ However, in cirrhotic human liver, ACE2 has been detected in most hepatocytes within the cirrhotic nodules. Additionally, hepatocytes cultured under hypoxic conditions have a significant increase of ACE2 expression at the mRNA level. The rate of expression increases in parallel to the duration of hypoxia. ${ }^{39}$ Likewise, hepatic steatosis seems to influence ACE-2 expression. In mice models of high fat diet-induced $\mathrm{NASH}$, increased hepatic ACE2 mRNA and protein expression have been observed, ${ }^{49}$ and this expression was even higher when animals were treated with PPARr agonists such as pioglitazone. ${ }^{49}$ While this phenomenon has not been shown in humans yet, this is an intriguing finding, since COVID-19 disease has been observed to be more severe in patients with diabetes and/or an elevated body mass index.

Studies have shown infection of hepatocytes by SARS$\mathrm{CoV},{ }^{9,50}$ from which the behavior of SARS-CoV-2 can be predicted (see - Fig. 2). Importantly, the presence of multiple

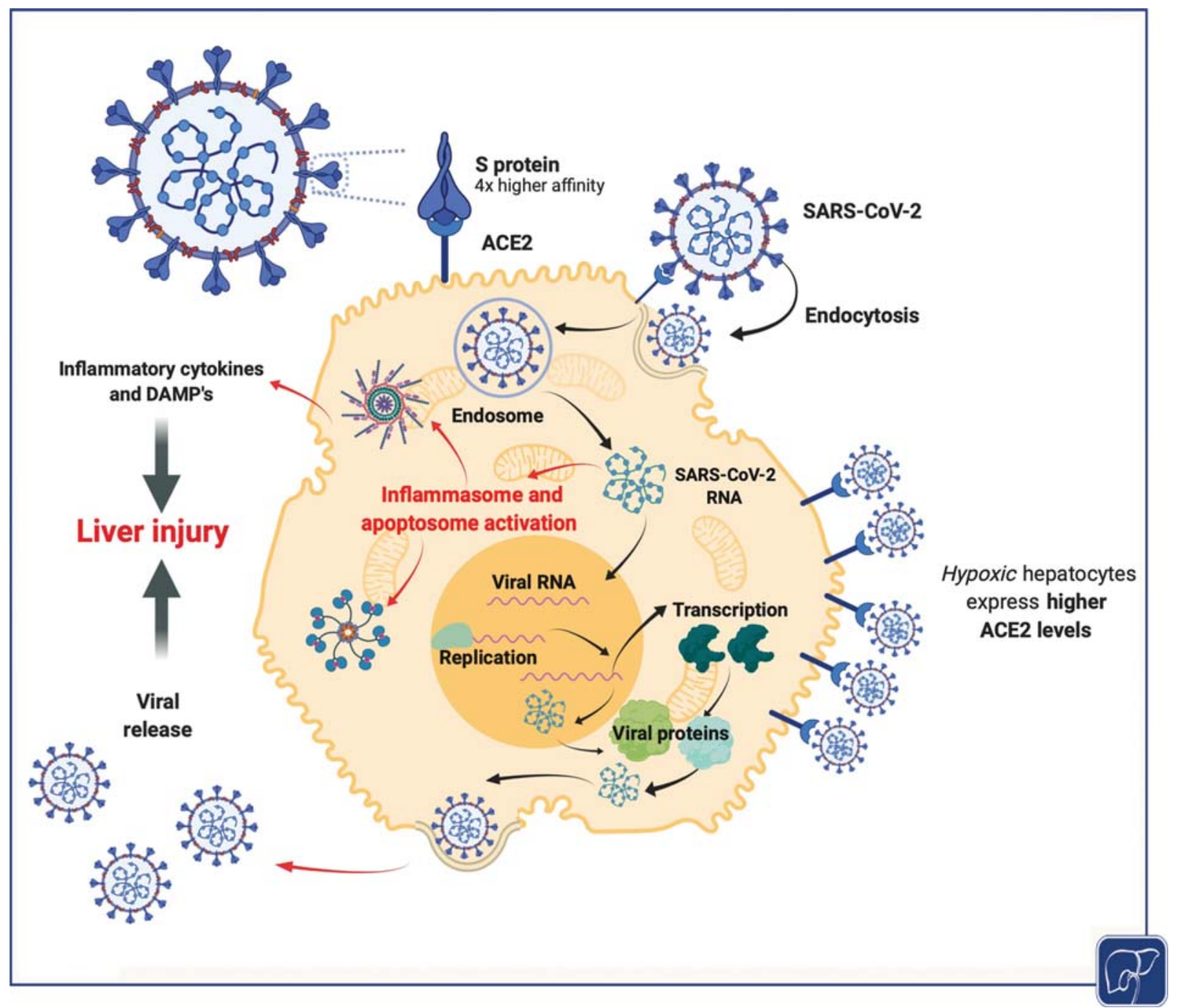

Fig. 2 Pathogenesis of severe acute respiratory syndrome coronavirus 2 (SARS-CoV-2) and possible mechanisms of hepatic injury. The targets of SARS-CoV-2 are angiotensin-converting enzyme 2 (ACE2) expressing cells. Current evidence demonstrates that hepatocytes are one of these target cells as the presence of viral particles in the cytoplasm has been observed. The viral internalization mainly occurs by endocytosis, although viral-host membrane fusion is also a possibility. This is mediated by the interaction of the S protein and ACE2. Liver injury can occur directly due to viral replication within hepatocytes; however, some of its virulence factors are known to function as inflammasome activators and proapoptotic proteins that induce the secretion of inflammatory cytokines, DAMPs, and hepatocyte apoptosis. On the contrary, the liver may suffer from the bystander effect and may respond and contribute to the inflammatory milieu, with consequences on the integrity of its hepatocytes. Interestingly, the hepatocyte may have a higher susceptibility for SARS-CoV-2 infection owing to the four times higher affinity of the S protein to ACE2, compared with SARS-CoV. It should also be considered that hepatocytes do not express ACE2 under normal conditions and that hypoxia related to severe inflammatory response syndrome or underlying liver disease may predispose them to infection by upregulating ACE2 expression, thus contributing to liver dysfunction. (BioRender was used to make the figures.) 
SARS-CoV-2 virions inside hepatocytes was recently demonstrated by transmission electron microscopy in autopsy liver tissue obtained from two patients with COVID-19. ${ }^{51}$ Microphotography also demonstrated mitochondrial swelling in the hepatocytes. Microvesicular steatosis, inflammation, and apoptotic bodies in the liver confirmed by TUNEL assay were also observed. ${ }^{51}$ Infection of liver tissue by SARS-CoV-2 has also been demonstrated through quantification of the ORF1ab and the $\mathrm{N}$ protein by qPCR in one out of four cases in a case series from Wuhan, China. ${ }^{43}$ While it is not clear from these studies whether SARS-CoV-2 gains entry into hepatocytes through the ACE2 receptor, ${ }^{51}$ it is known that SARS-CoV-2 possesses proteins like protein $7 \mathrm{a}$ and other nonstructural proteins which once inside the cells can induce apoptosis, ${ }^{9}$ such as that observed in these two patients.

Considering that COVID-19 patients suffer from severe hypoxia, and based on the earlier evidence indicating that hypoxic conditions upregulate the expression of ACE2 receptor on hepatocytes, one could argue that there is a big possibility that hepatocytes get directly infected by SARSCoV-2 and that such infection is mediated by ACE2 receptor. However, we cannot rule out the possibility of additional receptors used by SARS-CoV-2 to enter hepatocytes. With this limited evidence, we cannot definitely conclude that the presence of the virus inside hepatocytes indicates viral replication. But what is possible is that cell death may be caused by lymphocytes recognizing infected hepatocytes. ${ }^{52}$

SARS-CoV-2 and Immune-Mediated Collateral Damage Recognition of viruses through PRRs present on immune cells is crucial to halt the virus's life cycle, through the activation of mechanisms such as the inflammasome. ${ }^{29}$ On the contrary, the virulence factors are also capable of inducing a devastating SIRS. Indeed, SARS-CoV-2 has proteins like ORF3 and protein E that induce pyroptosis, a type of inflammatory cell death, by promoting the activation of the inflammasome in macrophages and lymphocytes which amplifies inflammatory signals. ${ }^{30,53}$

The high presence of macrophages and other immune cells in the liver suggests an increased susceptibility for the entrance of SARS-CoV-2 to this organ, and a possible mechanism for associated hepatic dysfunction. ${ }^{38}$ The liver may also contribute to the heightened inflammatory response, especially when injured. SARS-CoV-infected macrophages have demonstrated elevated levels of proinflammatory cytokines, which contribute to the pathogenesis of the dysregulated inflammatory response that is typical of SARS. ${ }^{54}$

Evidence suggests that the liver is one of the organs frequently affected by SARS-CoV and its associated SIRS. Reactive hepatitis as detected by elevated serum ALT levels was found in $24 \%$ of 294 SARS patients at admission and increased up to $69 \%$ during the disease course. ${ }^{55}$ Similarly, early hepatic dysfunction, defined as a serum bilirubin $\geq 33 \mu$ $\mathrm{mol} / \mathrm{L}$ ( $\geq 2 \mathrm{mg} / \mathrm{dL}$ ) was found with a prevalence of $17.6 \%$ in a cohort of 805 ARDS patients at admission, with significant increases in bilirubin levels observed during the clinical course of nonsurvivors. ${ }^{56}$

In a comparison analysis between SARS patients with normal and abnormal serum ALT levels, the latter had higher
IL-1 $\beta$, IL-6, and IL-10 levels. ${ }^{57}$ Higher IL-6 levels have been significantly associated with death in ARDS patients. ${ }^{58}$ Proinflammatory cytokines which are the mediators of hyperimmune responses induced by viral infections can lead to severe damage of different organs including the liver. Hepatocytes are known to be very susceptible to the different proinflammatory cytokines. When rat hepatocytes are subjected to septic stress and incubated with IL-1 $\beta$, overproduction of nitric oxide is enhanced, which results in mitochondrial dysfunction and consequent altered hepatocyte energy metabolism. ${ }^{59}$ IL-1 $\beta$ and IL- 6 are the main inducers of C-reactive protein (CRP), which is produced in the liver. ${ }^{60}$ Hepatic enzyme alterations in SARS-CoV-2-positive patients are also common and have been associated with the severity of the infection. ${ }^{48,61-64}$ Although CRP and IL-6 levels have been associated with higher mortality rates and severity of disease, ${ }^{65}$ reports in the literature are contradictory. Some studies have reported no difference in the elevation of proinflammatory biomarkers between patients with and without altered liver enzymes. ${ }^{51}$ Other studies have shown an association between abnormal LFTs and increased neutrophil counts ${ }^{51,66,67}$ and diminished lymphocytes. ${ }^{67}$ These patients also had a significantly longer hospital stay, ${ }^{51,66,68}$ severe lung injury as assessed by computed tomography or chest radiograph, ${ }^{51,66}$ and severe disease and admission to the intensive care unit (ICU). ${ }^{51,69} \mathrm{~A}$ similar pattern seems to occur in children, as higher total bilirubin (TBIL) and ALT levels were found in those with severe COVID19 disease, which also had elevated levels of inflammatory biomarkers such as IL-6, IL-10, CRP, and D-dimer, as well as diminished CD3 + T cell and NK cell counts. Nevertheless, only high TBIL levels and NK cell counts were identified by univariable analysis as the only significant risk factors associated with the development of severe pneumonia. ${ }^{65}$

Based on the aforementioned evidence, it is very possible that one of the mechanisms contributing to the damage observed in the liver of these patients is related to collateral damage produced by SARS-CoV-2-infected cells which upregulate the inflammasome and produce large amounts of cytokines to help combat the virus. However, this overactivation of the immune system may result in collateral damage to both infected and uninfected host cells stimulated either by the secreted cytokines or by the viral antigens. Additionally, in severe COVID-19 patients, this exacerbated systemic inflammatory response induces macro- and microcirculatory dysfunction that leads to a profound global hypoperfusion resulting in hypoxia, hypotension, and a hypercoagulable state. ${ }^{47}$ This has been noted in relevant histopathological findings from the liver of patients undergoing autopsy, where, besides important mononuclear infiltration, hepatocyte necrosis, vascular congestion, and thrombosis have been observed. ${ }^{47}$ Therefore, hypoxia, hypotension, and microvascular thrombosis should be considered important causes of liver injury/dysfunction in COVID-19 patients.

\section{Preexisting Liver Disease and COVID-19}

The liver is a vital organ. It is well perfused, being one of the only vital organs that have a portal system. As such, it is susceptible to injury when systemic inflammation ensues. 
Even though it has the capacity of compensatory hyperplasia, the circulating cytokines may very well inhibit this. Therefore, if the liver does not have enough functional reserve, when traumatic events such as ARDS and SIRS berate the body, liver failure may develop.

Initial data regarding whether preexisting liver disease was a predisposing factor for the development of liver injury and a more severe course indicated it was not. A meta-analysis considering nine single-center studies, the majority with more than 100 participants demonstrated that chronic liver disease was not significantly correlated with COVID-19 severity. However, it did show that patients with severe COVID-19 had a higher likelihood of developing acute liver dysfunction. ${ }^{70}$

Some single-center cross-sectional studies from China have shown no difference in the incidence of liver injury between ICU and non-ICU patients. ${ }^{6,12,66}$ Others have shown that only non-ICU patients had a history of liver disease; however, abnormal LFTs were present only in ICU patients. ${ }^{6,12}$

However, the most recent studies have now shown an important association between preexisting liver disease and COVID-19 severity. A retrospective study including 202 patients identified a higher prevalence of nonalcoholic fatty liver disease (NAFLD) in patients with severe COVID-19. Multivariate regression analysis showed patients with NAFLD were associated with COVID-19 progression (odds ratio, 6.4; 95\% confidence interval: 1.5-31.2). These patients also had higher likelihood of abnormal liver function from admission to discharge (70 vs. $11.1 \%, p<0.0001$ ) when compared with patients without NAFLD. However, there was no difference in the frequency of elevated enzymes between stable and severe COVID-19 infections. ${ }^{71}$ A multicenter study including 10 hospitals and a total of 5,771 patients demonstrated that chronic liver disease was more prevalent in patients with severe COVID-19 infection (1.2 vs. 2.1\%). The study also identified higher hepatic enzymes and bilirubin in patients with severe disease. ${ }^{67}$ Finally, data coming from a multicenter study including 37 health centers and more than 4 million participants in the United States identified a higher mortality and hospitalization rate in patients with preexisting liver disease. Preexisting liver disease conferred a relative risk (RR) of $3(1.5,6)$ for mortality and a RR of $1.3(1.1,1.6)$ for hospitalization. Although they did not calculate differences in the incidence of liver dysfunction between patients with preexisting liver disease and those without, both groups had a high number of patients with LFT abnormalities. ${ }^{72}$

Despite the conflicting data, overall the most recent information including a high number of patients indicates that preexisting liver disease confers a higher risk for developing severe COVID-19. Therefore, especial attention should be paid to patients with COVID-19 and preexisting liver diseases, considering the fact that there is evidence of an upregulation of ACE2 (97-fold in a widespread parenchymal pattern) in cirrhotic conditions, and in high-fat diet-induced NASH in animal models, ${ }^{49}$ treated with PPARr agonists such as pioglitazone ${ }^{49}$ which could increase susceptibility to SARS-CoV-2 infection. ${ }^{39}$ It should be considered that some patients may have subclinical preexisting liver disease due to the presence of metabolic syndrome. Of notice, glucose intolerance, metabolic syndrome, and diabetes are considered risk factors for a severe outcome in patients with COVID-19. ${ }^{73}$ The information described earlier together with the reports of liver dysfunction in SARS-CoV-2 severe cases $^{40}$ highlights the necessity to study this relation to implement early measures and prevent the permanent consequences due to devastating liver damage.

\section{Conclusion}

COVID-19 is caused by the SARS-CoV-2, whose viral structure is similar to that of SARS and MERS. However, SARSCoV-2 infection has been demonstrated to be more pathogenic due to unique properties of its $S$ protein. The infection was declared a pandemic in the beginning of the year, affecting more than 4 million people around the world. With a mortality rate of 3 to $4 \%$, the severity of the disease originates from the cytokine storm produced by the dysfunctional immune response to the virus and also due to the viruses' virulence factors. Liver injury develops in $36 \%$ of the patients, with mild to moderate elevation of hepatic enzymes. Recent studies highlighted in this review suggest that the injury is likely caused by the immune dysregulation, direct infection of the virus in liver parenchyma, and also by preexisting liver disease. Further research into the characterization of which mechanism predominates will help in prioritizing measures to prevent liver damage in patients with COVID-19.

\section{Main Concepts and Key Points}

- Liver injury has been observed in SARS-CoV-2-infected patients.

- Liver samples obtained from autopsies of COVID-19 patients show microvesicular steatosis, inflammation, vascular congestion, and thrombosis.

- Liver injury seen in COVID-19 disease may be due to direct cytopathic effect of the virus, immune-mediated collateral damage, and an exacerbation of preexisting liver disease.

- The liver is an organ likely to participate in the viral response against SARS-CoV-2 and this may predispose it to injury.

Funding

None.

Conflict of Interest

The authors declare no conflict of interest pertaining to this work.

\section{References}

1 ProMED. Undiagnosed pneumonia - China. Available at: https:// promedmail.org/promed-post/?id=6864153. Accessed May 1, 2020

2 World Health Organization. WHO Coronavirus Disease (COVID19) Dashboard. Available at: https://covid19.who.int. Accessed July 12,2020 
3 Centers for Disease Control and Prevention. Coronavirus (COVID19). Available at: https://www.cdc.gov/coronavirus/2019-ncov/index.html. Accessed May 1, 2020

$4 \mathrm{Wu} \mathrm{Z}, \mathrm{McGoogan}$ JM. Characteristics of and important lessons from the coronavirus disease 2019 (COVID-19) outbreak in China: summary of a report of 72314 cases from the Chinese Center for Disease Control and Prevention. JAMA 2020;323(13):1239-1242

5 McMichael TM, Currie DW, Clark S, et al; Public Health-Seattle and King County, EvergreenHealth, and CDC COVID-19 Investigation Team. Epidemiology of COVID-19 in a long-term care facility in King County, Washington. N Engl J Med 2020;382(21):2005-2011

6 Huang C, Wang Y, Li X, et al. Clinical features of patients infected with 2019 novel coronavirus in Wuhan, China. Lancet 2020;395 (10223):497-506

7 Mehta P, McAuley DF, Brown M, Sanchez E, Tattersall RS, Manson JJ; HLH Across Speciality Collaboration, UK. COVID-19: consider cytokine storm syndromes and immunosuppression. Lancet 2020;395(10229):1033-1034

8 Siu KL, Yuen KS, Castaño-Rodriguez C, et al. Severe acute respiratory syndrome coronavirus ORF3a protein activates the NLRP3 inflammasome by promoting TRAF3-dependent ubiquitination of ASC. FASEB J 2019;33(08):8865-8877

9 Chau TN, Lee KC, Yao H, et al. SARS-associated viral hepatitis caused by a novel coronavirus: report of three cases. Hepatology 2004;39(02):302-310

$10 \mathrm{Ng}$ DL, Al Hosani F, Keating MK, et al. Clinicopathologic, immunohistochemical, and ultrastructural findings of a fatal case of Middle East respiratory syndrome coronavirus infection in the United Arab Emirates, April 2014. Am J Pathol 2016;186(03): 652-658

11 Amanat F, Krammer F. SARS-CoV-2 vaccines: status report. Immunity 2020;52(04):583-589

12 Wang D, Hu B, Hu C, et al. Clinical characteristics of 138 hospitalized patients with 2019 novel coronavirus-infected pneumonia in Wuhan, China. JAMA 2020;323(11):1061-1069

13 Zhang C, Shi L, Wang F-S. Liver injury in COVID-19: management and challenges. Lancet Gastroenterol Hepatol 2020;5(05): 428-430

14 Zhou P, Yang XL, Wang XG, et al. A pneumonia outbreak associated with a new coronavirus of probable bat origin. Nature 2020;579 (7798):270-273

15 Yan R, Zhang Y, Li Y, Xia L, Guo Y, Zhou Q. Structural basis for the recognition of SARS-CoV-2 by full-length human ACE2. Science 2020;367(6485):1444-1448

16 Zhao Y, Zhao Z, Wang Y, Zhou Y, Ma Y, Zuo W Single-cell RNA expression profiling of ACE2, the receptor of SARS-CoV-2. Am J Respir Crit Care Med 2020. doi: 10.1164/rccm.202001-0179LE

17 Kim D, Lee J-Y, Yang J-S, Kim JW, Kim VN, Chang H. The architecture of SARS-CoV-2 transcriptome. Cell 2020;181(04):914-921.e10

18 Tai W, He L, Zhang X, et al. Characterization of the receptorbinding domain (RBD) of 2019 novel coronavirus: implication for development of RBD protein as a viral attachment inhibitor and vaccine. Cell Mol Immunol 2020;17(06):613-620

19 Ou X, Liu Y, Lei X, et al. Characterization of spike glycoprotein of SARS-CoV-2 on virus entry and its immune cross-reactivity with SARS-CoV. Nat Commun 2020;11(01):1620

20 Hoffmann M, Kleine-Weber H, Schroeder S, et al. SARS-CoV-2 cell entry depends on ACE2 and TMPRSS2 and is blocked by a clinically proven protease inhibitor. Cell 2020;181(02):271-280.e8

21 Shang J, Wan Y, Luo C, et al. Cell entry mechanisms of SARS-CoV-2. Proc Natl Acad Sci U S A 2020;117(21):11727-11734

22 Wang $\mathrm{Q}$ Z Zhang Y, Wu L, et al. Structural and functional basis of SARS-CoV-2 entry by using human ACE2. Cell 2020;181(04): 894-904.e9

23 Shang J, Ye G, Shi K, et al. Structural basis of receptor recognition by SARS-CoV-2. Nature 2020;581(7807):221-224

24 Recalcati S. Cutaneous manifestations in COVID-19: a first perspective. J Eur Acad Dermatol Venereol 2020;34(05):e212-e213
25 Castaño-Rodriguez C, Honrubia JM, Gutiérrez-Álvarez J, et al. Role of severe acute respiratory syndrome coronavirus viroporins $\mathrm{E}, 3 \mathrm{a}$, and 8a in replication and pathogenesis. MBio 2018;9(03):e02325-e17

26 Yang X, Yu Y, Xu J, et al. Clinical course and outcomes of critically ill patients with SARS-CoV-2 pneumonia in Wuhan, China: a single-centered, retrospective, observational study. Lancet Respir Med 2020;8(05):475-481

27 Meduri GU, Kohler G, Headley S, Tolley E, Stentz F, Postlethwaite A. Inflammatory cytokines in the BAL of patients with ARDS. Persistent elevation over time predicts poor outcome. Chest 1995; 108(05):1303-1314

28 Dolinay T, Kim YS, Howrylak J, et al. Inflammasome-regulated cytokines are critical mediators of acute lung injury. Am J Respir Crit Care Med 2012;185(11):1225-1234

29 Chen IY, Moriyama M, Chang M-F, Ichinohe T. Severe acute respiratory syndrome coronavirus viroporin $3 \mathrm{a}$ activates the NLRP3 inflammasome. Front Microbiol 2019;10:50

30 Fung S-Y, Yuen K-S, Ye Z-W, Chan C-P, Jin D-Y. A tug-of-war between severe acute respiratory syndrome coronavirus 2 and host antiviral defence: lessons from other pathogenic viruses. Emerg Microbes Infect 2020;9(01):558-570

31 Jose RJ, Manuel A. COVID-19 cytokine storm: the interplay between inflammation and coagulation. Lancet Respir Med 2020;8(06):e46-e47

32 Yang Z, Du J, Chen G, et al. Coronavirus MHV-A59 infects the lung and causes severe pneumonia in C57BL/6 mice. Virol Sin 2014;29 (06):393-402

33 Zalinger ZB, Elliott R, Weiss SR. Role of the inflammasome-related cytokines IL-1 and IL-18 during infection with murine coronavirus. J Neurovirol 2017;23(06):845-854

34 Lin SH, Fu J, Wang CJ, et al. Inflammation elevated IL-33 originating from the lung mediates inflammation in acute lung injury. Clin Immunol 2016;173:32-43

35 Chakraborty D, Zenker S, Rossaint J, et al. Alarmin S100A8 activates alveolar epithelial cells in the context of acute lung injury in a TLR4-dependent manner. Front Immunol 2017;8:1493

36 Sun S, Sursal T, Adibnia Y, et al. Mitochondrial DAMPs increase endothelial permeability through neutrophil dependent and independent pathways. PLoS One 2013;8(03):e59989

37 Heymann F, Tacke F. Immunology in the liver-from homeostasis to disease. Nat Rev Gastroenterol Hepatol 2016;13(02):88-110

38 Qi F, Qian S, Zhang S, Zhang Z. Single cell RNA sequencing of 13 human tissues identify cell types and receptors of human coronaviruses. Biochem Biophys Res Commun 2020;526(01):135-140

39 Paizis G, Tikellis C, Cooper ME, et al. Chronic liver injury in rats and humans upregulates the novel enzyme angiotensin converting enzyme 2. Gut 2005;54(12):1790-1796

40 Chen N, Zhou M, Dong X, et al. Epidemiological and clinical characteristics of 99 cases of 2019 novel coronavirus pneumonia in Wuhan, China: a descriptive study. Lancet 2020;395 (10223):507-513

41 Zippi M, Fiorino S, Occhigrossi G, Hong W. Hypertransaminasemia in the course of infection with SARS-CoV-2: incidence and pathogenetic hypothesis. World J Clin Cases 2020;8(08): 1385-1390

42 Barton LM, Duval EJ, Stroberg E, Ghosh S, Mukhopadhyay S. COVID-19 autopsies, Oklahoma, USA. Am J Clin Pathol 2020;153 (06):725-733

43 Tian S, Xiong Y, Liu H, et al. Pathological study of the 2019 novel coronavirus disease (COVID-19) through postmortem core biopsies. Mod Pathol 2020;33(06):1007-1014

44 Hanley B, Lucas SB, Youd E, Swift B, Osborn M. Autopsy in suspected COVID-19 cases. J Clin Pathol 2020;73(05):239-242

45 Chai X, Hu L, Zhang Y, et al. Specific ACE2 expression in cholangiocytes may cause liver damage after 2019-nCoV infection. bioRxiv2020. Doi: 10.1101/2020.02.03.931766

46 Zhao B, Ni C, Gao R, et al. Recapitulation of SARS-CoV-2 infection and cholangiocyte damage with human liver ductal organoids. 
Protein Cell 2020. Doi: 10.1007/s13238-020-00718-6 (Epub ahead of print)

47 Lax SF, Skok K, Zechner P, et al. Pulmonary arterial thrombosis in COVID-19 with fatal outcome: results from a prospective, singlecenter, clinicopathologic case series. Ann Intern Med 2020. Doi: 10.7326/m20-2566 (Epub ahead of print)

48 Zhang Y, Zheng L, Liu L, Zhao M, Xiao J, Zhao Q. Liver impairment in COVID-19 patients: a retrospective analysis of 115 cases from a single centre in Wuhan city, China. Liver Int 2020. Doi: 10.1111/ liv.14455 (Epub ahead of print)

49 Zhang W, Li C, Liu B, et al. Pioglitazone upregulates hepatic angiotensin converting enzyme 2 expression in rats with steatohepatitis. Ann Hepatol 2013;12(06):892-900

50 Ding Y, He L, Zhang Q et al. Organ distribution of severe acute respiratory syndrome (SARS) associated coronavirus (SARS-CoV) in SARS patients: implications for pathogenesis and virus transmission pathways. J Pathol 2004;203(02):622-630

51 Wang Y, Liu S, Liu H, et al. SARS-CoV-2 infection of the liver directly contributes to hepatic impairment in patients with COVID-19. J Hepatol 2020;S0168-8278(20):30294-4

52 Maini MK, Boni C, Lee CK, et al. The role of virus-specific CD8(+) cells in liver damage and viral control during persistent hepatitis B virus infection. J Exp Med 2000;191(08):1269-1280

$53 \mathrm{Xu}$ J, Zhao S, Teng T, et al. Systematic comparison of two animalto-human transmitted human coronaviruses: SARS-CoV-2 and SARS-CoV. Viruses 2020;12(02):244

54 Channappanavar R, Perlman S. Pathogenic human coronavirus infections: causes and consequences of cytokine storm and immunopathology. Semin Immunopathol 2017;39(05):529-539

55 Chan HL, Kwan AC, To KF, et al. Clinical significance of hepatic derangement in severe acute respiratory syndrome. World J Gastroenterol 2005;11(14):2148-2153

56 Dizier S, Forel J-M, Ayzac L, et al; ACURASYS Study Investigators; PROSEVA Study Group. Early hepatic dysfunction is associated with a worse outcome in patients presenting with acute respiratory distress syndrome: a post-hoc analysis of the ACURASYS and PROSEVA studies. PLoS One 2015;10(12):e0144278

57 Duan ZP, Chen Y, Zhang J, et al. [Clinical characteristics and mechanism of liver injury in patients with severe acute respiratory syndrome]. Zhonghua Gan Zang Bing Za Zhi 2003;11(08):493-496

$58 \mathrm{Wu}$ C, Chen X, Cai Y, et al. Risk factors associated with acute respiratory distress syndrome and death in patients with coronavirus disease 2019 pneumonia in Wuhan, China. JAMA Intern Med 2020; Doi: 10.1001/jamainternmed.2020.0994 (Epub ahead of print)

59 Tu W, Satoi S, Zhang Z, et al. Hepatocellular dysfunction induced by nitric oxide production in hepatocytes isolated from rats with sepsis. Shock 2003;19(04):373-377
60 Sproston NR, Ashworth JJ. Role of C-reactive protein at sites of inflammation and infection. Front Immunol 2018;9:754

61 Yang F, Shi S, Zhu J, Shi J, Dai K, Chen X. Analysis of 92 deceased patients with COVID-19. J Med Virol 2020;15(Apr):. Doi: 10.1002/ jmv.25891

62 Jin X, Lian JS, Hu JH, et al. Epidemiological, clinical and virological characteristics of 74 cases of coronavirus-infected disease 2019 (COVID-19) with gastrointestinal symptoms. Gut 2020;69(06): 1002-1009

$63 \mathrm{Wu}$ J, Liu J, Zhao X, et al. Clinical characteristics of imported cases of COVID-19 in Jiangsu Province: a multicenter descriptive study. Clin Infect Dis 2020; Doi: 10.1093/cid/ciaa199 (Epub ahead of print)

64 Li X, Wang L, Yan S, et al. Clinical characteristics of 25 death cases with COVID-19: A retrospective review of medical records in a single medical center, Wuhan, China. Int J Infect Dis 2020; 94:128-132

65 Wang Y, Zhu F, Wang C, et al. The risk of children hospitalized with severe COVID-19 in Wuhan. Pediatr Infect Dis J 2020;39(07): e91-e94

66 Xie H, Zhao J, Lian N, Lin S, Xie Q Zhuo H. Clinical characteristics of non-ICU hospitalized patients with coronavirus disease 2019 and liver injury: a retrospective study. Liver Int 2020;40(06): 1321-1326

67 Lei F, Liu YM, Zhou F, et al. Longitudinal association between markers of liver injury and mortality in COVID-19 in China. Hepatology 2020. Doi: 10.1002/hep.31301 (Epub ahead of print)

68 Fan Z, Chen L, Li J, et al. Clinical features of COVID-19-related liver damage. Clin Gastroenterol Hepatol 2020;18(07): 1561-1566

69 Cai Q Huang D, Yu H, et al. COVID-19: abnormal liver function tests. J Hepatol 2020:S0168-8278(20)30218-X

70 Wang X, Fang X, Cai Z, et al. Comorbid chronic diseases and acute organ injuries are strongly correlated with disease severity and mortality among COVID-19 patients: a systemic review and metaanalysis. Research (Wash D C) 2020;2020:2402961

71 Ji D, Qin E, Xu J, et al. Non-alcoholic fatty liver diseases in patients with COVID-19: a retrospective study. J Hepatol 2020:S01688278(20)30206-3

72 Singh S, Khan A. Clinical characteristics and outcomes of COVID19 among patients with pre-existing liver disease in United States: a multi-center research network study. Gastroenterology 2020. Doi: 10.1053/j.gastro.2020.04.064 (Epub ahead of print)

73 Zhu L, She Z-G, Cheng X, et al. Association of blood glucose control and outcomes in patients with COVID-19 and pre-existing type 2 diabetes. Cell Metab 2020;31(06):1068-1077.e3 